\title{
Exclusive breastfeeding promotion by peer counsellors in sub-Saharan Africa (PROMISE-EBF): a cluster-randomised trial
}

Thorkild Tylleskar, Debra J ackson, Nicolas Meda, Ingunn Marie S Engebretsen, Mickey Chopra, Abdoulaye Hama Diallo, Tanya Doherty, Eva-Charlotte Ekstrom, Lars T Fadnes, Ameena Goga, Chipepo Kankasa, J ern I Klungsoyr, Carl Lombard, Victoria Nankabirwa, J olly K Nankunda, Philippe Van de Perre, David Sanders, Rebecca Shanmugam, Halvor Sommerfelt, Henry Wamani, J ames KTumwine, for the PROMISE-EBF Study Group

\section{Summary}

Background: Exclusive breastfeeding (EBF) is reported to be a life-saving intervention in low-income settings. The effect of breastfeeding counselling by peer counsellors was assessed in Africa.

Methods: 24 communities in Burkina Faso, 24 in Uganda, and 34 in South Africa were assigned in a 1:1 ratio, by use of a computer-generated randomisation sequence, to the control or intervention clusters. In the intervention group, we scheduled one antenatal breastfeeding peer counselling visit and four post-delivery visits by trained peers. The data gathering team were masked to the intervention allocation. The primary outcomes were prevalance of EBF and diarrhoea reported by mothers for infants aged 12 weeks and 24 weeks. Country-specific prevalence ratios were adjusted for cluster effects and sites. Analysis was by intention to treat. This study is registered with ClinicalTrials.gov, number NCT00397150.

Findings: 2579 mother-infant pairs were assigned to the intervention or control clusters in Burkina Faso ( $n=392$ and $n=402$, respectively), Uganda ( $n=396$ and $n=369$, respectively), and South Africa ( $\mathrm{n}=535$ and 485 , respectively). The EBF prevalences based on 24-h recall at 12 weeks in the intervention and control clusters were 310 (79\%) of 392 and 139 (35\%) of 402, respectively, in Burkina Faso (prevalence ratio 2-29, 95\% CI 1-33-3-92); 323 (82\%) of 396 and 161 (44\%) of 369, respectively, in Uganda (1-89, 1-70-2-11); and 56 (10\%) of 535 and 30 (6\%) of 485, respectively, in South Africa (1-72, 1-12-2-63). The EBF prevalences based on 7-day recall in the intervention and control clusters were 300 (77\%) and 94 (23\%), respectively, in Burkina Faso (3-27, 2-13-5-03); 305 (77\%) and 125 (34\%), respectively, in Uganda (2-30, 2-00-2-65); and 41 (8\%) and 19 (4\%), respectively, in South Africa (1-98, 1-30-3-02). At 24 weeks, the prevalences based on 24-h recall were 286 (73\%) in the intervention cluster and 88 (22\%) in the control cluster in Burkina Faso (3-33, 1-74-6-38); 232 (59\%) and 57 (15\%), respectively, in Uganda (3-83, 2-97-4-95); and 12 (2\%) and two (<1\%), respectively, in South Africa (5-70, 1-33-24-26). The prevalences based on 7-day recall were $279(71 \%)$ in the intervention cluster and 38 (9\%) in the control cluster in Burkina Faso (7-53, 4-42-12-82); 203 (51\%) and 41 (11\%), respectively, in Uganda (4-66, 3-35-6-49); and ten (2\%) and one (<1\%), respectively, in South Africa (9-83, 1-40-69-14). Diarrhoea prevalence at age 12 weeks in the intervention and control clusters was 20 (5\%) and 36 (9\%), respectively, in Burkina Faso (0-57, 0-27-1-22); 39 (10\%) and 32 (9\%), respectively, in Uganda (1-13, 0-81-1-59); and 45 (8\%) and 33 (7\%), respectively, in South Africa (1-16, 0-78-1-75). The prevalence at age 24 weeks in the intervention and control clusters was 26 
(7\%) and 32 (8\%), respectively, in Burkina Faso (0-83, 0-45-1-54); 52 (13\%) and 59 (16\%), respectively, in Uganda (0-82, 0-58-1-15); and 54 (10\%) and 33 (7\%), respectively, in South Africa (1-31, 0-89-1-93).

Interpretation: Low-intensity individual breastfeeding peer counselling is achievable and, although it does not affect the diarrhoea prevalence, can be used to effectively increase EBF prevalence in many sub-Saharan African settings.

Funding: European Union Sixth Framework International Cooperation-Developing Countries, Research Council of Norway, Swedish International Development Cooperation Agency, Norwegian Programme for Development, Research and Education, South African National Research Foundation, and Rockefeller Brothers Foundation.

\section{Introduction}

Promotion of exclusive breastfeeding (EBF) for the first 6 months of life has been estimated to be the most effective preventive strategy for saving the lives of young children in low-income settings, 1 and could contribute towards the Millennium Development Goal 4 of reducing child mortality. High coverage of promotion and support of breastfeeding could prevent 22 million (8-6\%) disability adjusted life years.2

WHO recommends EBF for 6 months. ${ }^{3}$ However, in sub-Saharan Africa, initiatives to promote EBF are hampered by the HIV epidemics. ${ }^{4}$ Only one randomised facility-based study has been done in Ghana; 5 no trials of community-based EBF promotion had been undertaken in sub-Saharan Africa at the time our trial was initiated. A recent review of breastfeeding promotion summarised the small amount of evidence from Africa. ${ }^{6}$ In the past decade, most studies in which EBF was promoted in sub-Saharan Africa had been undertaken within the framework of prevention of mother-to-child transmission of HIV 1 to assess whether EBF could increase HIV-free survival. ${ }^{4}$ The results indicated that intensive interventions could lead to near-universal adherence to EBF. Investigation of whether less intensive community-based interventions can achieve substantial benefits in feeding patterns is important.7

PROMISE-EBF is the first trial undertaken in Africa to assess the effect of individual home-based EBF peer counselling. We report here the results of the analysis of the primary outcomes in Burkina Faso, Uganda, and South Africa only because severe floods midway through the study site in Zambia disrupted the data gathering.

\section{Methods Study sites}

The study was undertaken in rural Banfora, southwest Burkina Faso, Mbale District, eastern Uganda, and Paarl (a periurban site close to Cape Town), Umlazi (periurban site near Durban), and Rietvlei (rural Kwa Zulu Natal), South Africa. Further details of the study sites are provided in the webappendix pp 2-7).

\section{Study design}

PROMISE-EBF was a multicentre community-based cluster-randomised behavioural-intervention trial that was done in sub-Saharan Africa (webappendix pp 2-7). To assess the potential for scale up of the EBF intervention, we randomly assigned clusters rather than mother-infant pairs, thereby avoiding the potential contamination between intervention and control groups, and more accurately mimicking an intervention that is to be undertaken on a full 
scale. Peer counsellors provided breastfeeding support in intervention clusters. Mothers and infants in control clusters in Burkina Faso and Uganda were given standard health care only, and those control clusters in South Africa were visited by peer counsellors, with the same schedule as in the intervention clusters, but who assisted families in obtaining birth certificates and social welfare grants. The peer counsellors for the intervention and control clusters in South Africa were kept separate during the study.

Inclusion in the study was a two-stage process, starting with the assessment of pregnant women (preinclusion) and then the inclusion of the mother- infant pairs (inclusion). Pregnant women intending to breastfeed were assessed for study participation, after being identified by community-based recruiters (for further details, see webappendix pp 8-9). Almost all mothers in the clusters were reached, irrespective of birth place. The preinclusion criteria were that the woman resided in the selected cluster; was 7 months or visibly pregnant; had no plans to move in the forthcoming year; and provided informed consent. At the 3-week post-partum assessment a preincluded mother-infant pair was included if there was a singleton livebirth with no severe malformation that could interfere with breastfeeding. Exclusion criteria for the women were severe psychological illness, which could interfere with consent and study participation; giving birth more than 1 week before preinclusion; or a plan to replacement feed. Unless a clear reason for non- participation in a scheduled visit was given, three attempts to visit the mother-infant pair were made before a visit was judged to be missed and this increased the number of untimely visits. A recruited mother was revisited until the last scheduled visit, irrespective of the number of missed visits, unless there was a clear reason for termination.

\section{Randomisation and masking}

We used simple computer-generated randomisation, with clusters assigned in a 1:1 allocation ratio. The allocation sequences for Burkina Faso and Uganda were generated by HS and Rajiv Bahl, and for South Africa by CL. HS, Rajiv Bahl, and CL also assigned clusters to the trial groups. In Burkina Faso, randomisation was not stratified; in Uganda, it was stratified according to urban and rural location, and in South Africa according to the three sites. Clusters were mapped based on criteria of accessibility, population size, health system, and health data. Within each stratum, the clusters were randomly assigned to intervention or control on the basis of numbers generated in Excel 97. Only the data collectors were masked to the allocation assignment-ie, they were not informed about the cluster allocation and were kept separate from the intervention team; they enrolled the eligible participants. The success of this masking was not formally assessed. Further details about the randomisation and masking are provided in the webappendix $\mathrm{p}$ 8). Because of fairly large clusters in Burkina Faso and South Africa, all eligible women in the intervention clusters were enrolled in the study for peer counselling, whereas a randomly selected subset were approached for data gathering. The recruitment interview was scheduled antenatally or within 1 week after birth. Visits for data gathering were scheduled at weeks 3, 6, 12, and 24 after birth. We defined timely visits (9-18 weeks for the visit at 12 weeks, and 18-28 weeks for the visit at 24 weeks) to be included in the prevalence estimates for EBF and diarrhoea. The figure shows the total and timely visits. In all countries, peer support was offered to all women in intervention clusters irrespective of whether they were enrolled for data gathering, and women were approached for data gathering irrespective of whether they agreed to participate in peer-support visits, consistent with a community-based study for measurement of the effect of an intervention when undertaken on full scale. 
Study approval was obtained by the institutional review board of Centre Muraz (number 013/2005/ CE-CM) and by the Ministry of Health at national and regional levels in Burkina Faso; Faculty of Medicine, Makerere University, and Research and Ethics Committee, Uganda National Council for Science and Technology; ethics committee for the Medical Research Council, South Africa; and regional committees for Medical and Health Research Ethics in Norway. Women provided verbal informed consent for participation in the peer-counselling programme, which was regarded as a service, whereas signed or thumb-printed informed consent was obtained before data were gathered. 

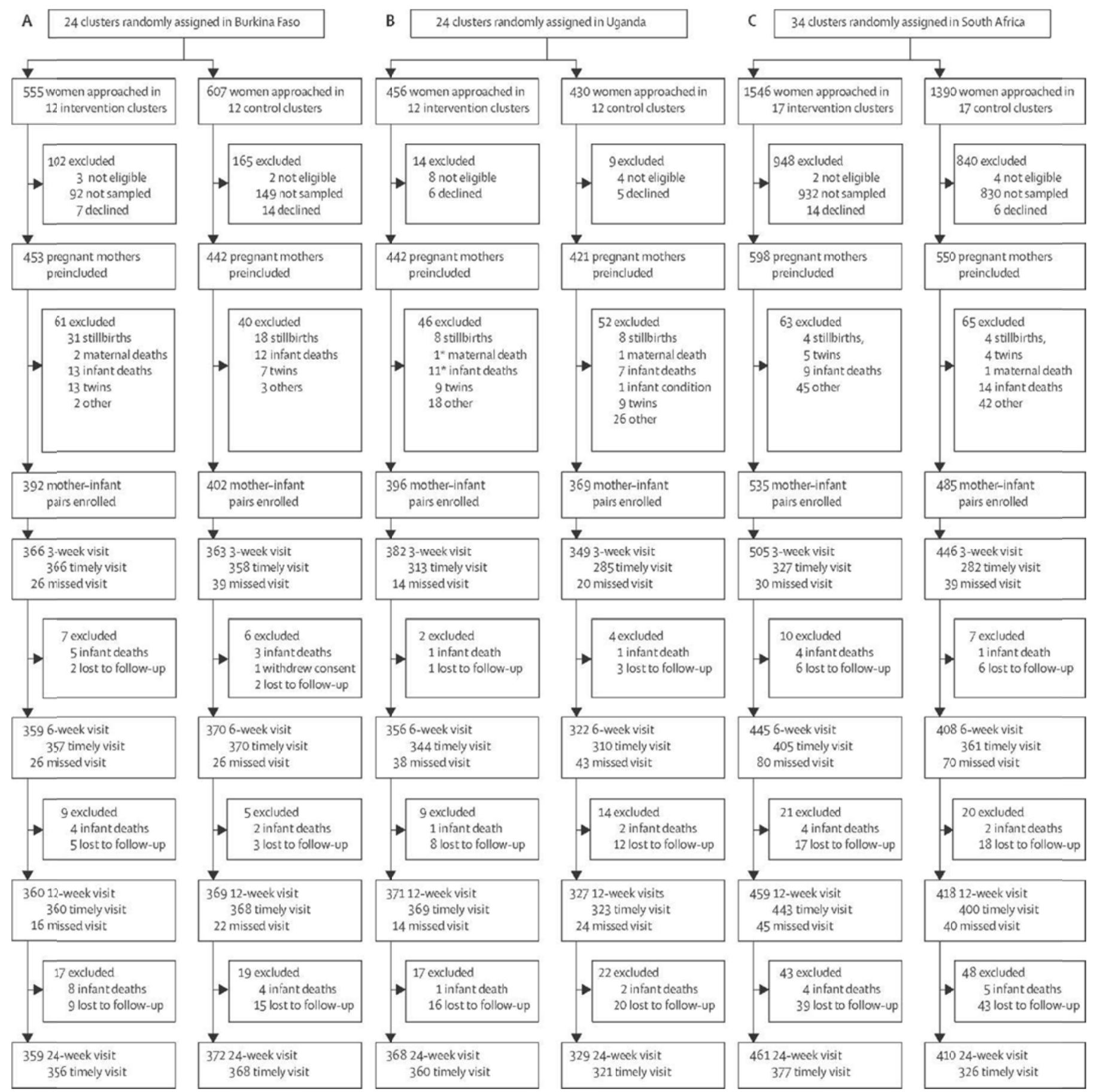

Figure: Trial profile

(A) Burkina Faso. (B) Uganda. (C) South Africa. *One infant death and one maternal death combined. 


\section{Peer counselling}

We recruited peer counsellors who were living in or near the intervention clusters. Peer counsellors were identified and trained for 1 week by the national research teams. The counsellors offered home-based-breastfeeding peer support to the mothers in the intervention clusters. Peer counsellors received continuous mentoring (fortnightly to monthly) by study-appointed supervisors. All mothers were offered at least five visits, starting with a visit in the third trimester. In Burkina Faso, mothers were scheduled to have home visits during the first week postnatally, and thereafter at weeks 2, 4, 8, 16, and 20. In Uganda and South Africa, home visits were scheduled within the first week and thereafter at weeks 4,7 , and 10 . Detailed descriptions of the intervention are provided in the webappendix pp 12-14 and elsewhere.810 The course material was based on the WHO courses: Breastfeeding counselling: a training course, and HIV and infant feeding counselling: a training course. The courses were integrated and adapted at sites for local circumstances. The intervention was piloted in Uganda. ${ }^{11}$ Peer counsellors provided information, and encouraged and supported EBF for 6 months. Mothers with any breastfeeding difficulties were referred to a health worker with training in lactation management (Burkina Faso and Uganda) or to a public health provider who was aware of the HIV status of the participant (South Africa).

\section{Data capture and management}

Study questionnaires were developed and adapted from Bland and colleagues ${ }^{12}$ and Piwoz, ${ }^{13}$ and previous work undertaken in participating countries. 14-17 The questionnaires were piloted in Uganda in 2005.18 Data were gathered in the mothers' homes from 2006 to 2008 by trained data gatherers. In Burkina Faso and Uganda, data were gathered with hand-held computers by use of EpiHandy (version 165.528-142 RC; webappendix p 15). In South Africa, the data were gathered by use of paper questionnaires that were entered by use of EpiData (version 3.1).

\section{Definitions}

Current breastfeeding was assessed at all scheduled post- partum visits with past 24-h and 7-day recalls. Additionally, dietary recalls consisted of 22 specified items and one open question. Babies who did not receive any other food or liquids other than breast milk were classified as exclusively breastfed, even if they had been administered drugs. ${ }^{13}$ Prevalence of diarrhoea was based on the mothers' reports of the past 2 weeks. A socioeconomic index was created with Multiple Correspondence Analysis (Stata SE, version 11.0), based on a set of country-specific assets that included house type and toilet facilities. ${ }^{19}$ Every country-specific index was divided into quintiles.

\section{Statistical analysis}

Sample size calculation was done independently for the two primary outcomes EBF and diarrhoea at ages 12 weeks and 24 weeks, with 95\% CIs and 80\% power. The cluster sizes corresponded to an estimated average of 35 deliveries per year. The coefficient of variation between the clusters used in the sample size calculation was 0-4 for EBF prevalence. To detect an increase from $20 \%$ to $40 \%$ in EBF prevalence in each country, we needed a minimum of 12 clusters per group per country. For the diarrhoea prevalence sample size calculation, the coefficient of variation was assumed to be $0-3$. To detect a $33 \%$ reduction in diarrhoea 
prevalence from $12 \%$ to $8 \%$, a total of 48 clusters in each group were needed. 106 clusters-34 in South Africa, 24 in Burkina Faso, 24 in Uganda, and 24 in Zambia-were randomly assigned. To compensate for the loss of the Zambian site, we combined the observations at 12 weeks and 24 weeks of infant age to obtain an adequate precision for effects of the intervention on diarrhoea prevalence.

All eligible mother-infant pairs were analysed with Stata SE (version 11.0). The models of EBF and diarrhoea were country specific. We calculated prevalence ratios for EBF and diarrhoea with a generalised linear model for the binomial family with a log link taking the design effect and stratification into account. Additionally, for diarrhoea only, we used Poisson regression to estimate the event ratio between the intervention and the control groups of the trial, combining the number of reported periods of 2 weeks with diarrhoea at age 12 weeks and 24 weeks, in a pooled estimate of Burkina Faso, Uganda, and South Africa. This variable could accordingly have the values 0,1 , or 2 .

The analysis presented in this report included all mother- infant pairs in the denominator, implying that we recoded missing, lost to follow-up, and death as non-events-ie, not having exclusively breastfed or not having had diarrhoea. We also undertook an analysis excluding individuals without valid information (webappendix pp 21-22).

This study is registered with ClinicalTrials.gov, number NCT00397150.

\section{Role of the funding source}

The sponsor of the study had no role in study design, data collection, data analysis, data interpretation, or writing the report. The corresponding author had full access to all the data in the study and had final responsibility for the decision to submit for publication. 


\begin{tabular}{|c|c|c|c|c|c|c|}
\hline & \multicolumn{2}{|l|}{ Burkina Faso } & \multicolumn{2}{|l|}{ Uganda } & \multicolumn{2}{|l|}{ South Africa } \\
\hline & Intervention & Control & Intervention & Control & Intervention & Control \\
\hline \multicolumn{7}{|l|}{ Categorical data } \\
\hline Eligible mother-infant pairs & 392 & 402 & 396 & 369 & 535 & 485 \\
\hline Marital status & 390 & 402 & 392 & 366 & 533 & 484 \\
\hline Married & $333(85 \%)$ & $351(87 \%)$ & $244(62 \%)$ & $234(64 \%)$ & $134(25 \%)$ & $114(24 \%)$ \\
\hline Cohabiting & $32(8 \%)$ & $34(8 \%)$ & $119(30 \%)$ & $104(28 \%)$ & $36(7 \%)$ & $30(6 \%)$ \\
\hline Single, widowed, separated, or divorced & $25(6 \%)$ & $17(4 \%)$ & $29(7 \%)$ & $28(8 \%)$ & $363(68 \%)$ & $340(70 \%)$ \\
\hline Socioeconomic-status quintile & 392 & 401 & 396 & 369 & 535 & 485 \\
\hline 1 (poorest) & $82(21 \%)$ & $81(20 \%)$ & $91(23 \%)$ & $62(17 \%)$ & $111(21 \%)$ & $85(18 \%)$ \\
\hline 2 & $77(20 \%)$ & $77(19 \%)$ & $97(24 \%)$ & $86(23 \%)$ & $100(19 \%)$ & $87(18 \%)$ \\
\hline 3 & $76(19 \%)$ & $85(21 \%)$ & $76(19 \%)$ & $49(13 \%)$ & $103(19 \%)$ & $100(21 \%)$ \\
\hline 4 & $76(19 \%)$ & $85(21 \%)$ & $71(18 \%)$ & $84(23 \%)$ & $111(21 \%)$ & $113(23 \%)$ \\
\hline 5 (least poor) & $81(21 \%)$ & $73(18 \%)$ & $61(15 \%)$ & $88(24 \%)$ & $110(21 \%)$ & $100(21 \%)$ \\
\hline Electricity in house & 389 & 402 & 391 & 361 & 535 & 485 \\
\hline Yes & $18(5 \%)$ & $5(1 \%)$ & $53(14 \%)$ & $70(19 \%)$ & $409(77 \%)$ & $445(92 \%)$ \\
\hline Water source & 388 & 400 & 392 & 363 & 535 & 484 \\
\hline Surface water and other & $117(30 \%)$ & $161(40 \%)$ & $136(35 \%)$ & $97(27 \%)$ & $121(23 \%)$ & $105(22 \%)$ \\
\hline Borehole or tap & $271(70 \%)$ & $238(60 \%)$ & $244(62 \%)$ & $246(68 \%)$ & $68(13 \%)$ & $58(12 \%)$ \\
\hline Piped yardor home & 0 & $1(<1 \%)$ & $12(3 \%)$ & $20(6 \%)$ & $346(65 \%)$ & $321(66 \%)$ \\
\hline Toilet & 168 & 232 & 339 & 328 & 515 & 456 \\
\hline None oropen & $165(98 \%)$ & $229(99 \%)$ & $84(25 \%)$ & $59(18 \%)$ & $82(16 \%)$ & $67(15 \%)$ \\
\hline Pit or ventilated improved pit & $3(2 \%)$ & $3(1 \%)$ & $245(72 \%)$ & $266(81 \%)$ & $262(51 \%)$ & $238(52 \%)$ \\
\hline Flush & 0 & 0 & $10(3 \%)$ & $3(<1 \%)$ & $171(33 \%)$ & $151(33 \%)$ \\
\hline Parity & 391 & 402 & 392 & 366 & 535 & 485 \\
\hline Primipara & $64(16 \%)$ & $65(16 \%)$ & $81(21 \%)$ & $85(23 \%)$ & $261(49 \%)$ & $247(51 \%)$ \\
\hline Multipara & $327(84 \%)$ & $337(84 \%)$ & $311(79 \%)$ & $281(77 \%)$ & $274(51 \%)$ & $238(49 \%)$ \\
\hline Previouschild death & 325 & 337 & 307 & 277 & 395 & 362 \\
\hline Yes & $190(58 \%)$ & $201(60 \%)$ & $109(36 \%)$ & $80(29 \%)$ & $29(7 \%)$ & $23(6 \%)$ \\
\hline Attendance at antenatal clinic (index child) & 389 & 401 & 376 & 352 & 532 & 481 \\
\hline Yes & $284(73 \%)$ & $285(71 \%)$ & $272(72 \%)$ & $274(78 \%)$ & $527(99 \%)$ & $470(98 \%)$ \\
\hline Place of birth (index child) & 372 & 370 & 381 & 351 & 514 & 461 \\
\hline Out of facility & $229(62 \%)$ & $241(65 \%)$ & $208(55 \%)$ & $146(42 \%)$ & $28(5 \%)$ & $38(8 \%)$ \\
\hline Facility & $143(38 \%)$ & $128(35 \%)$ & $173(45 \%)$ & $205(58 \%)$ & $486(95 \%)$ & $423(92 \%)$ \\
\hline \multicolumn{7}{|l|}{ Continuous data } \\
\hline Maternal age & 292 & 318 & 394 & 368 & 533 & 485 \\
\hline Years & $25(20-30)$ & $25(20-30)$ & $25(20-30)$ & $24(20-30)$ & $23(19-29)$ & $23(19-28)$ \\
\hline Maternal education & 377 & 379 & 391 & 365 & 535 & 485 \\
\hline Years & $0(0-0)$ & $0(0-0)$ & $6(4-8)$ & $6(5-9)$ & $10(9-12)$ & $10(9-12)$ \\
\hline Maternal body-mass index & 247 & 214 & 343 & 312 & 432 & 398 \\
\hline At 6 weeks $\left(\mathrm{kg} / \mathrm{m}^{2}\right)^{*}$ & $21(20-22)$ & $22(20-23)$ & $22(20-24)$ & $22(20-24)$ & $26(23-29)$ & $25(22-30)$ \\
\hline Monthly income & 318 & 330 & 116 & 121 & 508 & 452 \\
\hline 2007 euros & $3(2-8)$ & $3(3-8)$ & $14(5-28)$ & $10(5-23)$ & $101(63-158)$ & $105(63-162)$ \\
\hline
\end{tabular}




\section{Results}

Overall, 4984 women were approached for participation in the study from May, 2006, to J uly, 2008 (figure). The final sample for data gathering and analysis was 2579 mother infant pairs. Few baseline differences were noted between the intervention and control clusters at the country level (table 1). Burkina Faso had the poorest participants with the lowest education, whereas the participants from South Africa were the richest with the highest education.

In all three countries, prevalence of EBF at 12 weeks of age in the intervention cluster was about twice that in the control cluster (table 2) whether based on a 24-h or 7-day recall of feeding practices. The prevalence ratios for EBF obtained with the 24 -h and 7-day recalls were also similar (table 2). The prevalence of EBF was lower at 24 weeks of age than at 12 weeks in the intervention and control clusters in Burkino Faso, Uganda, and South Africa, whereas the differences (prevalence ratios) were higher (table 2).

No differences were noted for the prevalence of diarrhoea at age 12 weeks or 24 weeks between the clusters within the countries (data not shown) or all the clusters (prevalence ratio 0.95, 95\% CI $0 \cdot 71-1.26$, at 12 weeks, and $0.96,0 \cdot 75-1 \cdot 23$, at 24 weeks; table 3). The ratio of the prevalence of reported diarrhoea for 2 weeks at age 12 weeks and 24 weeks in the intervention versus the control clusters in all three countries was $0 \cdot 95(0 \cdot 78-1 \cdot 17)$.

Similar results were obtained when the analysis with missing information set as non-events was compared with the analysis omitting subjects with missing information (webappendix pp 21- 22). Socioeconomic status did not confound the associations in any of the countries (data not shown).

\section{Discussion}

Overall, peer counselling was an effective strategy to increase the practice of EBF in Burkina Faso, Uganda, and South Africa. This effect was particularly important in Burkina Faso and Uganda. At infant age of 12 weeks, about a third of mothers breastfed exclusively in the control clusters in these two countries compared with about $80 \%$ in the intervention clusters. The situation in South Africa was quite different with EBF being rare at baseline, and even though the intervention also had a significant effect, the absolute increase was small. Prevalence of diarrhoea before 12 weeks and 24 weeks of age was not significantly different between the intervention and control clusters between these three countries.

The findings of this study are similar to those of similar intervention studies in other parts of the world (panel). Frequent counselling has been associated with a large effect on EBF practices, including two larger studies in Bangladesh ${ }^{21}$ and India, ${ }^{22}$ and a gradient between counselling frequency and EBF prevalences was shown in Mexico. ${ }^{23}$ The results of our study are also similar to those of a meta-analysis of breastfeeding interventions ${ }^{2}$ in which individual counselling nearly doubled any breastfeeding at age 6 months. The results of the metaanalysis of breastfeeding promotion suggest that facility- based group counselling has a larger effect than does individual counselling. This strategy might, however, not reach women who rarely use health facilities. 


\begin{tabular}{|c|c|c|c|c|c|c|}
\hline & \multicolumn{3}{|l|}{12 weeks } & \multicolumn{3}{|l|}{24 weeks } \\
\hline & Intervention & Control & $\begin{array}{l}\text { Prevalence ratio } \\
(95 \% \text { Cl) }\end{array}$ & Intervention & Control & $\begin{array}{l}\text { Prevalence ratio } \\
(95 \% \text { CI) }\end{array}$ \\
\hline \multicolumn{7}{|c|}{ Burkina Faso } \\
\hline 24-h recall & $310 / 392(79 \%)$ & $139 / 402(35 \%)$ & $2.29(1.33-3.92)$ & $286 / 392(73 \%)$ & $88 / 402(22 \%)$ & $3.33(1.74-6.38)$ \\
\hline 7-day recall & $300 / 392(77 \%)$ & $94 / 402(23 \%)$ & $3.27(2.13-5.03)$ & $279 / 392(71 \%)$ & $38 / 402(9 \%)$ & $7 \cdot 53(4 \cdot 42-12 \cdot 82)$ \\
\hline \multicolumn{7}{|l|}{ Uganda } \\
\hline 24-h recall & $323 / 396(82 \%)$ & $161 / 369(44 \%)$ & $1.89(1.70-2.11)$ & $232 / 396(59 \%)$ & $57 / 369(15 \%)$ & $3.83(2.97-4.95)$ \\
\hline 7-day recall & $305 / 396(77 \%)$ & $125 / 369(34 \%)$ & $2.30(2.00-2.65)$ & $203 / 396(51 \%)$ & $41 / 369(11 \%)$ & $4 \cdot 66(3 \cdot 35-6 \cdot 49)$ \\
\hline \multicolumn{7}{|c|}{ South Africa } \\
\hline 24-h recall & $56 / 535(10 \%)$ & $30 / 485(6 \%)$ & $1.72(1.12-2 \cdot 63)$ & $12 / 535(2 \%)$ & $2 / 485(<1 \%)$ & $5.70(1 \cdot 33-24 \cdot 26)$ \\
\hline 7-day recall & $41 / 535(8 \%)$ & $19 / 485(4 \%)$ & $1.98(1.30-3.02)$ & $10 / 535(2 \%)$ & $1 / 485(<1 \%)$ & $9.83(1.40-69 \cdot 14)$ \\
\hline
\end{tabular}

\begin{tabular}{|c|c|c|c|c|c|c|}
\hline & \multicolumn{3}{|l|}{12 weeks } & \multicolumn{3}{|l|}{24 weeks } \\
\hline & Intervention & Control & $\begin{array}{l}\text { Prevalence ratio } \\
(95 \% \text { CI) }\end{array}$ & Intervention & Control & $\begin{array}{l}\text { Prevalence ratio } \\
(95 \% \text { Cl) }\end{array}$ \\
\hline Burkina Faso & $20 / 392(5 \%)$ & $36 / 402(9 \%)$ & $0.57(0.27-1.22)$ & $26 / 392(7 \%)$ & $32 / 402(8 \%)$ & $0.83(0.45-1.54)$ \\
\hline Uganda & $39 / 396(10 \%)$ & $32 / 369(9 \%)$ & $1.13(0.81-1.59)$ & $52 / 396(13 \%)$ & $59 / 369(16 \%)$ & $0.82(0.58-1.15)$ \\
\hline South Africa & $45 / 535(8 \%)$ & $33 / 485(7 \%)$ & $1.16(0.78-1.75)$ & $54 / 535(10 \%)$ & $33 / 485(7 \%)$ & $1.31(0.89-1.93)$ \\
\hline
\end{tabular}

The contexts of the countries need to be taken into account during the interpretation of the findings of our trial. Rural and urban diets are similar in South Africa, with most people in rural farming areas purchasing most of their food, whereas subsistence farming is common in rural Uganda and Burkina Faso. Furthermore, the South African Department of Health's routine child health services have a history of promoting commercial infant formula as part of the protein energy malnutrition scheme. ${ }^{24}$ Additionally, the national programme for prevention of mother-to-child transmission of HIV-1 provided free formula to HIV-infected mothers. Furthermore, the International Code of Marketing of Breast-milk Substitutes ${ }^{25}$ is not yet legislated in South Africa, allowing the uncontrolled marketing of infant formula throughout the country. Mixed messages are likely to undermine EBF.26 Burkina Faso and Uganda do not provide free formula through the health system and EBF is much more common, as suggested by the data from our control communities in these two countries.

\section{Panel: Research in context Systematic review}

We searched PubMed up to April 26, 2011, with the search terms "Randomized Controlled Trial" and Breast Feeding*, and "Health Promotion/methods*" for randomised trials in which exclusive breastfeeding (EBF) was promoted in low-income countries, and with Breast Feeding* only for reviews published in the past 5 years. EBF has been widely promoted in other contexts by use of facility-based group counselling, individual counselling, community 
outreach, and campaigns with differing effects on EBF acceptance and practice. A recent meta-analysis ${ }^{2}$ of 30 studies (of which none was done in Africa) showed a positive effect of EBF on breastfeeding at 6 months of age (odds ratio 1-93, 95\% CI 1-18-3-15). Diarrhoea has been the most frequently reported child health outcome related to breastfeeding peer counselling. In a review ${ }^{6}$ done in 2010, five randomised trials were identified in which diarrhoea prevalence or incidence was reduced by peer support promotion of EBF. Two studies were undertaken in Latin America, two in Asia, and one in Africa. The African trial was a smaller facility-based trial of diarrhoea re-occurrence. In another African trial, in Guinea-Bissau, ${ }^{20}$ no effect of peer counselling was noted on diarrhoea incidence in a birth cohort that was followed up for 6 months (Poisson regression rate ratio 0-99 (0-84-1-17). In this study, breastfeeding was widely practised and the intervention delayed the introduction of other foods and drinks.

\section{Interpretation}

The results of PROMISE EBF show that EBF promotion by peer counsellors more than doubled the proportion of mothers who reported to have exclusively breastfed their infants. Our results for diarrhoea prevalence are similar to those of the trial in Guinea-Bissau. Moreover, epidemiological, behavioural, and other contextual differences are likely to vary greatly between different population groups and, although too early to draw conclusions about the effect of EBF promotion on diarrhoea morbidity from EBF promotion in sub-Saharan Africa as a whole, one hypothesis would be that a shift from a predominantly breastfed infant population to exclusively breastfed does not reduce the diarrhoea-associated morbidity.

Results of qualitative studies associated with our trial showed a high degree of satisfaction with the intervention among mothers in Uganda ${ }^{10}$ and Burkina Faso (data not shown). By contrast, in South Africa, $, 9,27$ targeted concerted actions seem to be needed. These include support to mothers while ensuring that marketing of formula milk is regulated and that health systems harmonise their approaches to infant feeding to avoid giving mothers conflicting messages.

We accordingly undertook a pooled analysis across these three countries that, with a high precision, showed that the intervention did not affect diarrhoea prevalence. In a cluster randomised trial in Guinea-Bissau, a similarly high precision showed that delayed introduction of water and weaning foods had no effect on diarrhoeal morbidity. ${ }^{20}$

However, breastfeeding might have less effect on the incidence than on the duration and severity of diarrhoea. Our 2-week prevalence assessment includes incident episodes and episodes that started just before but continued into the 2-week observation. So although diarrhoeal duration contributed to our diarrhoeal disease burden estimate, it was not measured as a separate entity. Notably, in a cluster-randomised trial in India, with

roughly the same effect on the prevalence of reported EBF, promotion of EBF substantially reduced the prevalence of diarrhoea. ${ }^{22}$ In an observational prospective study in Bangladesh, babies exclusively breastfed in the first few months of life had an almost four times lower risk of diarrhoeal deaths than did those who received no breast milk or were partly breastfed. ${ }^{28}$ 
In communities where predominant breastfeeding is common, such as Guinea-Bissau, ${ }^{20}$ and Burkina Faso and Uganda with mainly predominant breastfeeding, a shift to EBF might not generate large differences in diarrhoeal morbidity or mortality. In a multicountry cohort study, although not being breastfed was associated with a substantially increased diarrhoeal mortality risk, babies who were exclusively breastfed were not at a lower risk of dying from diarrhoea than were those predominantly breastfed. ${ }^{29}$

Our trial was a large community-based and cluster- randomised intervention with an intervention-intensity appropriate for scale-up within national health services. The community-based approach could possibly have resulted in socially desirable answers, and the results were based on self-reports. A bias towards desirable answers and thereby an increased effect size cannot be ruled out. However, the very large effects on reported EBF are unlikely to be attributable to information bias. We also noted some questionnaire fatigue in the Ugandan site-ie, reluctance to fully engage in answering similar questions after a few interviews. ${ }^{18}$

Overall, a fairly low-intensity peer-counselling intervention to promote EBF for infants aged up to 6 months in Burkina Faso, Uganda, and South Africa resulted in an increase in EBF prevalence ratios at age 12 weeks and 24 weeks. With the success of increasing EBF prevalence elsewhere, ${ }^{2}$ our findings suggest that the prevalence of EBF can be increased in many sub-Saharan African settings. However, the intervention did not lead to a reduction in diarrhoeal prevalence, and additional studies are needed to assess its effect on other child health and development outcomes.

From a policy-perspective, to achieve the Millennium Development Goal 4, when initiating interventions with specially trained community peers interacting with parents before and after delivery about infant-feeding counselling, the opportunity to undertake other interventions to reduce maternal and neonatal mortality that target mother-infant pairs during pregnancy and early childhood should not be missed-at least not in the 68 countdown countries ${ }^{30}$ where maternal and infant mortality remains high.

\section{Contributors}

TT, DJ , NM, IMSE, MC, TD, AG, CK, JIK, JKN, PVdP, DS, HS, HW, and J KT designed the study and intervention. TT, DJ , IMSE, MC, AHD, TD, E-CE, JIK, CL, HS, and J KT elaborated the data gathering methods and database architecture. TT, DJ, NM, IMSE, MC, AHD, TD, E-CE, J IK, VN, J KN, HS, and J KT implemented the study at the different sites. TT, DJ , IMSE, AHD, TD, LTF, J IK, CL, VN, RS, HS, and HW did the data management and statistical analysis. All authors, except MC, CK, and JIK, were active in the interpretation of the results. The report was prepared by TT, DJ , and IMSE, and reviewed by AHD, TD, LTF, AG, CL, VN, J KN, PVdP, DS, and HS. All authors have approved the final report.

\section{The PROMISE-EBF Study Group}


Steering Committee: Thorkild Tylleskar, Philippe Van de Perre, Eva-Charlotte Ekstrom, Nicolas Meda, J ames K Tumwine, Chipepo Kankasa, Debra J ackson.

Participating countries and investigators: Norway Thorkild Tylleskar*, Ingunn M S Engebretsen, Lars T Fadnes, Eli Fjeld Falnes, Knut Fylkesnes, J Orn I KlungsOyr, Anne Nordrehaug-Astr0m, Bjarne Robberstad, Halvor Sommerfelt; France Philippe Van de Perre*; Sweden Eva-Charlotte Ekstrom*, Barni Nor; Burkina Faso Nicolas Meda*, Abdoulaye Hama Diallo, Thomas Ouedrago, J eremi Rouamba, Bernadette Traore Germain Traore, Emmanuel Zabsonre; Uganda J ames K Tumwine*, Caleb Bwengye, Charles Karamagi, Victoria Nankabirwa, J olly K Nankunda, Grace Ndeezi, Margaret Wandera; Zambia Chipepo Kankasa*, Mary Katepa-Bwalya, Chafye Siuluta, Seter Siziya; South Africa Debra J ackson*, Mickey Chopra, Mark Colvin, Tanya Doherty, Ameena Goga, Lungiswa Nkonki, David Sanders, Wesley Solomon, Wanga Zembe. *For every country, principal investigators are listed first, other authors are listed in alphabetical order of surname.

\section{Conflicts of interest}

We declare that we have no conflicts of interest.

\section{Acknowledgments}

The study was funded by the European Union Sixth Framework International Cooperation-Developing Countries (contract number INCO-CT 2004-003660); Research Council of Norway; Swedish International Development Cooperation Agency; Norwegian Programme for Development, Research and Education; Rockefeller Brothers Foundation; and the South African National Research Foundation. We thank the mothers and their families for their contributions to this study; representative study teams for their efforts; local health providers and administrative local councils for their cooperation; and Rajiv Bahl, WHO, for the assistance in planning the study and in the randomisation.

\section{References}

1 J ones G, Steketee RW, Black RE, Bhutta ZA, Morris SS, and the Bellagio Child Survival Study Group. How many child deaths can we prevent this year? Lancet 2003; 362: 65-71. 2 Bhutta ZA, Ahmed T, Black RE, et al, for the Maternal and Child Undernutrition Study Group. What works? Interventions for maternal and child undernutrition and survival. Lancet 2008; 371: 417-40.

3 Kramer MS, Kakuma R. Optimal duration of exclusive breastfeeding. Cochrane Database Syst Rev 2002; 1: CD003517

4 Coovadia HM, Bland RM. Preserving breastfeeding practice through the HIV pandemic. Trop Med Int Health 2007; 12: 1116-33.

5 Aidam BA, Perez-Escamilla R, Lartey A. Lactation counseling increases exclusive breast-feeding rates in Ghana. J Nutr 2005; 135: 1691-95.

6 Chapman DJ , Morel K, Anderson AK, Damio G, Perez-Escamilla R. Breastfeeding peer counseling: from efficacy through scale-up.

J Hum Lact 2010; 26: 314-26. 
Bhandari N, Kabir AK, Salam MA. Mainstreaming nutrition into maternal and child health programmes: scaling up of exclusive breastfeeding. Matern Child Nutr2008; 4 (suppl 1): 5-23. \& Nankunda J, Tylleskar T, Ndeezi G, Semiyaga N, Tumwine JK. Establishing individual peer counselling for exclusive breastfeeding in Uganda: implications for scaling-up. Matern Child Nutr 2010; 6: 53-66.

9 Nor B, Zembe Y, Daniels K, et al. "Peer but not peer": considering the context of infant feeding peer counseling in a high HIV prevalence area. J Hum Lact 2009; 25: 427-34.

10 Nankunda J, Tumwine J K, Nankabirwa V, Tylleskar T, Study Group PE. "She would sit with me": mothers' experiences

of individual peer support for exclusive breastfeeding in Uganda. Int Breastfeed J 2010; 5: 16.

${ }_{11}$ Nankunda J, Tumwine J K, Soltvedt A, Semiyaga N, Ndeezi G, Tylleskar T. Community based peer counsellors for support of exclusive breastfeeding: experiences from rural Uganda. Int Breastfeed J 2006; 1: 19.

${ }_{12}$ Bland RM, Rollins NC, Solarsh G, Van den Broeck J, Coovadia HM. Maternal recall of exclusive breast feeding duration. Arch Dis Child 2003; 88: 778-83.

${ }_{13}$ Piwoz EG. Breastfeeding and replacement feeding practices in the context of mother-to-child transmission of HIV. An assessment tool for research. WH0/RHR/ 01.12, WH0/CAH/ 01.21. Geneva, Switzerland: World Health Organization, Department of Reproductive Health and Research (RHR), Department of Child and Adolescent Health and Development, 2001.

${ }_{14}$ Doherty T, Chopra M, Jackson D, Goga A, Colvin M, Persson LA. Effectiveness of the WHO/ UNICEF guidelines on infant feeding for HIV-positive women: results from a prospective cohort study in South Africa. AIDS 2007; 21: 1791-977

${ }_{15}$ Engebretsen IM, Wamani H, Karamagi CA, Semiyaga N, Tumwine JK, Tylleskar T. Low adherence to exclusive breastfeeding in Eastern Uganda: a community-based cross-sectional study comparing dietary recall since birth with 24-hour recall.

BMC Pediatr 2007; 7: 10.

${ }_{16}$ Fjeld E, Siziya S, Katepa-Bwalya M, Kankasa C, Moland KM, Tylleskar T. 'No sister, the breast alone is not enough for my baby'

a qualitative assessment of potentials and barriers in the promotion of exclusive breastfeeding in southern Zambia. Int Breastfeed J 2008; 3: 26.

${ }_{17}$ Goga AE, Van Wyk B, Doherty T, Colvin M, J ackson DJ, Chopra M. Operational effectiveness of guidelines on complete breast-feeding cessation to reduce mother-to-child transmission of HIV: results from a prospective observational cohort study at routine prevention of mother-to-child transmission sites, South Africa.

J Acquir Immune Defic Syndr 2009; 50: 521-28.

${ }_{18}$ Engebretsen IM, Shanmugam R, Sommerfelt AE, Tumwine JK, Tylleskar T. Infant feeding modalities addressed in two different ways in Eastern Uganda. Int Breastfeed J 2010; 5: 2. 19 Howe LD, Hargreaves J R, Huttly SR. Issues in the construction of wealth indices for the measurement of socio-economic position in low-income countries. Emerg Themes Epidemiol 2008; 5: 3. 
${ }_{20}$ J akobsen MS, Sodemann M, Biai S, Nielsen J, Aaby P. Promotion of exclusive breastfeeding is not likely to be cost effective in West Africa. A randomized intervention study from Guinea-Bissau. Acta Paediatr 2008; 97: 68-75.

${ }_{21}$ Haider R, Ashworth A, Kabir I, Huttly SR. Effect of community-based peer counsellors on exclusive breastfeeding practices in Dhaka, Bangladesh: a randomised controlled trial. Lancet 2000; 356: 1643^-7

22 Bhandari N, Bahl R, Mazumdar S, Martines J, Black RE, Bhan MK, and the other members of the Infant Feeding Study Group. Effect of community-based promotion of exclusive breastfeeding on diarrhoeal illness and growth: a cluster randomised controlled trial. Lancet 2003; 361: 1418-23.

23 Morrow AL, Guerrero ML, Shults J, et al. Efficacy of home-based peer counselling to promote exclusive breastfeeding: a randomised controlled trial. Lancet 1999; 353: 1226-31.

${ }_{24}$ Hendricks MK, Roux ML, Fernandes M, Irlam J. Evaluation of a nutrition supplementation programme in the Northern Cape Province of South Africa. Public Health Nutr 2003; 6: 431-37

${ }_{25}$ The International Code of Marketing of Breast-Milk Substitutes: frequently asked questions. Geneva: World Health Organization, 2008.

26 Doherty T, Sanders D, Goga A, J ackson D. Implications of the new WHO guidelines on HIV and infant feeding for child survival in South Africa. Bull World Health Organ 2011; 89: 62-67 ${ }_{27}$ Daniels K, Nor B, Jackson D, Ekstrom EC, Doherty T. Supervision of community peer counsellors for infant feeding in South Africa: an exploratory qualitative study. Hum Resour Health 2010; 8: 6.

${ }_{28}$ Arifeen S, Black RE, Antelman G, Baqui A, Caulfield L, Becker S. Exclusive breastfeeding reduces acute respiratory infection and diarrhea deaths among infants in Dhaka slums.

Pediatrics; 108: E67

29 Bahl R, Frost C, Kirkwood BR, et al. Infant feeding patterns and risks of death and hospitalization in the first half of infancy: multicentre cohort study. Bull World Health Organ 2005; 83: 418-26.

30 Bhutta ZA, Chopra M, Axelson H, et al. Countdown to 2015 decade report (2000-10): taking stock of maternal, newborn, and child survival. Lancet 2010; 375: 2032-44. 